\title{
A Study of Basalt Fingers Using Experiments and Numerical Simulations in Double-diffusive Systems
}

\author{
O. P. Singh (Corresponding author) \\ Department of Mechanical Engineering, Indian Institute of Science, Bangalore-560 012, India \\ Tel: 918-870-275-490Ｅ-mail: om.prakashh.singh@gmail.com \\ D. Ranjan (Corresponding author) \\ Department of Mechanical Engineering, Texas A\&M University, USA \\ Tel: 979-458-3580 E-mail: dranjan@tamu.edu
}

\section{J. Srinivasan}

Centre for Atmospheric and Oceanic Sciences, Indian Institute of Science, Bangalore-560 012, India

Tel: 918-022-933-068 E-mail: jayes@caos.iisc.ernet.in

K. R. Sreenivas

Engineering Mechanics Unit,

Jawaharlal Nehru Centre for Advanced Scientific Research, Bangalore-560 064. India

Tel: 918-022-082-836 E-mail: krs@jncasr.ac.in

Received: October 8, 2010 Accepted: October 29, 2010 doi:10.5539/jgg.v3n1p42

\begin{abstract}
This study examined the evolution of columnar-basalt structures using simple laboratory experiments and numerical simulation in double-diffusive finger system. Effect of various parameters like Prandtl and Rayleigh numbers has been considered in the study. Columnar-basalts are geological formations observed in some of the ponded basalt-lava found at many parts of the world. They are prismatic rock joints having polygonal cross-section with straight edges and parallel faces. The typical cross-sectional dimensions vary from few centimeters to meter size and typical height from 30-50 meters. Hence the aspect ratio of the columnar-basalt in most cases is greater than 100. The limitations for the evolution of columnar basalts provided in the literature have been discussed. An order of magnitude analysis has been presented for the thickness of the unstable layer at the interface. In this paper, we demonstrate that the double diffusive finger convection system can produce features similar to those observed in columnar basalt and can serve as an alternative, plausible explanation for the evolution of columnar basalt structure.
\end{abstract}

Keywords: Double-diffusive convection, Columnar-basalt, Rayleigh number, Density stability ratio, Viscosity

\section{Introduction}

Double diffusive convection has fascinated many researchers over the years because of its wide application in the various fields of astrophysics, chemistry, geology, metallurgy, meteorology, oceanography, growth of semiconductor crystals (Schmitt, 1983; Huppert and Sparks, 1984; Platten and Legros 1984). Columnar joints are spectacular geological phenomena observed in many parts of the world and are formed by solidification of the basaltic lava. Columnar structures have straight edges and parallel faces, they generally have polygonal cross-section with typical dimension varying from few centimeters to meters size. The column height is much larger than their cross sectional size, typical aspect ratio of 100 is common. In Figure 1, we show some of the famous columnar-basaltic structures found around the world. Columnar joints are rarely seen in thin lava flows and are common in thick flows ponded in depressions (Kantha, 1981). Gravity is an important factor since most 
of the columnar joints have their longer axis parallel to the gravity vector. In many places well-developed columnar structures (colonnade) are sandwiched between two irregular, fractured columnar regions with variety of shapes (entablature). Any model proposed for explaining columnar basalt evolution should reproduce the observed high aspect ratio and sandwich configuration in these structures. In most of the cases composition is uniform across the section of a column, but there are exceptions in which both color and the composition vary across the cross section of a column (Kantha, 1981). Another important feature of the columnar-basalt structure is the rarity of its occurrence across the globe.

Sosman (1916) proposed that hexagonal structures in columnar basalt are caused by convection cells (similar to the one observed in Bs nard-convection) in the molten lava before solidification. Sosman's model was based on experiments with wax and oil in a flat dish. This model cannot be accepted as an explanation for the evolution of columnar-basalt structures since the aspect ratio for the Benard-convection-cell is around one, which is much lower than that observed in columnar-basalt. Spry (1962) suggested that molten lava contracts as it solidifies and cracks form due to thermal stresses. There are many experimental studies on stress induced fragmentation, for example, Groisman and Kaplan (1994), Muller (1998), Shorlin et al. (2000), and Pauchard (2003). In this set of experiments, researchers study the formation of polygonal cracks when slurry is desiccated. Main observations from these experiments are (a) width and the number of sides of the polygonal crack depends on the layer thickness, (b) morphology and the crack pattern depend on crack nucleation sites, and (c) width also depends on physical properties of the slurry. Pauchard et al. (2003) showed that in a homogeneous medium crack pattern tend to be irregular whereas a medium with coarse-grains would produce regular "columnar cracks".

These experimental studies indicate following limitations of the Spry's model for explaining the evolution of columnar basalt structures. From the experimental results of Pauchard et al. (2003), in a brittle and continuous material like basalt, cracks are unlikely to produce regular structures as observed in the columnar basalt. Secondly, Muller's experiments do not relate physical properties of the basalt and the observed size of the columnar-basalt, which could then explain rarity of columnar basalt structures among many basaltic lava flows around the world. Spry's model does not explain the unique "fluid-like" shapes in the entablature. Finally, in directional crack propagation experiments, crack first appears from the surface and propagates into the medium, this will not explain observed sandwich configuration of columnar basalts.

Kantha $(1980,1981)$ has argued that the slender, finger-like convection cells formed in a double diffusive system could represent columnar-basalt. Convection in a double diffusive system is due to the diffusion of two components having different molecular diffusivities (e.g. heat and salt) with opposing contributions to the vertical density distribution in a fluid medium. In a two-layer double diffusive system (Figure 3a), when the top layer has an excess concentration of a heavier species (with a smaller diffusion coefficient than the heat) and hotter than the bottom layer, long finger like convection cells will evolve. These convection cells, due to their slender shape, are known as double diffusive fingers (DDF). Cross sectional shapes of these fingers are polygons (Shirtcliffe and Turner, 1970) and the aspect ratio is high. The importance of double diffusive convection in geological processes has been highlighted by many investigators (Turner, 1979; Griffiths, 1986; Huppert and Sparks, 1984; and Kerr et. al., 1989). The role of double diffusive finger convection in the evolution of columnar-basalt has not been studied much so far.

If lava flows into a region and is topped by another batch of lava after some time, which has a higher temperature and is richer in the concentration of a heavier species than the bottom layer, then the interaction between these two batches of lava can produce double diffusive fingers. Kantha $(1980,1981)$ highlighted many morphological similarities between DDF and columnar basalt structures. Some of the important similarities between these structures are (a) high aspect ratio in the DDF and columnar basalt, (b) polygonal cross sectional shapes and (c) sandwich configuration with chaotic structure in the entablature and columnar structure in the colonnade with similarities to DDF morphology in salt-heat two-layer experiments. Based on these similarities he speculated that double-diffusive convection in the molten lava pool could have generated basalt-fingers. The grain boundaries between the rising and falling legs of the basalt finger-convection cells could have served as natural path for crack propagation in the solidified lava. Kantha was unable to show how a DDF with a typical cross-sectional dimension of $1-2 \mathrm{~mm}$ in the laboratory heat-salt experiments can scale up to $10-80 \mathrm{~cm}$ as observed in columnar basalt. One of the relevant factors that would contribute to the increase in the cross sectional dimension of a DDF is the high viscosity of lava. Kantha conducted double-diffusive experiments with glycerin-water mixture as the working medium and salt and sugar as two diffusing components to demonstrate the effect of viscosity on the finger width. However, for the reasons, which will be stated in the later part of this paper, his experiment did not show a marked effect of viscosity on the width of the fingers. Hence the DDF as a possible mechanism for the formation of columnar basalt has not been proven so far. 
In this paper we present results from experiments (section 2) and numerical simulations (section 3) to demonstrate the possibility of getting thick fingers, similar to columnar basalt structures, using high viscosity fluids. Results and discussions are presented in section 4. An order of magnitude estimates for the density differences that are needed in the double-diffusive columnar basalts evolution has been presented. Conclusions are presented in section 5 .

\section{Experimental procedure and results}

We carried out a series of laboratory experiments to study the effect of viscosity on the double-diffusive finger evolution. Experiments were performed in a transparent, glass tank having glass walls ( $5 \mathrm{~mm}$ thick). The inner dimension of the tank was $19 \mathrm{~cm} \times 19 \mathrm{~cm} \times 19 \mathrm{~cm}$. An air gap of $5 \mathrm{~mm}$ was provided around all the sides (for insulation) by placing an outer glass tank. We performed the experiments in a thermo-haline double diffusive system (heat and salt as two diffusing components in water). Sodium carboxy-methyl cellulose (CMC) powder was used for enhancing the viscosity of the water, $\mathrm{CMC}$ will increases the viscosity of the water without affecting other properties (Davaille, 1999). The concentration of CMC in the solution was varied from $0.05 \%$ to $0.5 \% \mathrm{CMC}$ by weight.

Flow visualization is useful technique to qualitatively and quantitatively study finger convection. We used a non-intrusive visualization technique in which a water-soluble fluorescent dye is used as a scalar flow marker. In this work, fluorescein sodium dye is added to the upper layer fluid and, then, made to fluoresce by passing a sheet of light through the one plane of the tank (see Figure 2). The flow field was illuminated by a thin sheet of light obtained by passing a focused 250 watts slide projection light beam through a slit. The slit was made by placing two sharp-edged blades side by side in a slide holder. The effective width of the light sheet was 2-3 mm at the focused position and this was found to produce acceptable light levels and contrast. Once the initial step like state was established (Figure 3a), the initially static system was allowed to evolve. We recorded the evolution of convection using a $35 \mathrm{~mm}$ high-resolution camera. All the photographs of vertical cross-section of the finger structures were taken from a direction normal to the light sheet.

Two snapshots of the vertical cross-section of the double diffusive system are given in Figure 3(b, c). Figure $3 b$ shows the condition just after the establishment of the two layer double diffusive system and Figure $3 \mathrm{c}$ provides a snapshot after the development of salt fingers. The remarkable features of this experiment (at high viscosity) with pure water heat/salt or salt /sugar experiments are (i) complete absence of horizontal convection in adjoining layers and (ii) evolution of thick and regularly spaced fingers. These salt fingers penetrate all through the reservoirs keeping their structure intact until they reach the bottom plate of the setup. We observed from the series of experiments conducted with different viscosity fluids that, a high aspect ratio, thick finger can be generated for a double-diffusive system in a fluid having high viscosity.

\section{Numerical model}

In this section we examine the effect of fluid viscosity (or Prandtl number, Pr) and other parameters on the width of the DDF using two-dimensional numerical simulations. The faster diffusing component (denoted as $T$ ) and slower diffusing component (denoted as $\mathrm{S}$ ) govern the convection phenomenon where density is influenced by both the components. Density of the fluid, $\rho=\rho_{0}\left(1-\beta_{\mathrm{T}} \mathrm{T}+\beta_{\mathrm{S}} \mathrm{S}\right)$, where $\rho_{0}$ is the reference density; $\beta_{\mathrm{T}}$ and $\beta_{\mathrm{S}}$ are the volumetric expansion coefficients for temperature and concentration. Non-dimensional governing equations for momentum transport, energy and species with continuity has the following form (refer to Turner, 1979; Shen and Veronis, 1997) and they were solved numerically by Patankar's (1980) finite volume method.

$$
\nabla \cdot u=0
$$

$$
\begin{gathered}
\frac{\partial u}{\partial t^{*}}+(u . \nabla) u=-\nabla p+\operatorname{Pr} \nabla^{2} u-\left[\operatorname{Pr} R a_{T}\left(\frac{S^{*}}{R_{\rho}}-T^{*}\right)\right] k \\
\frac{\partial T^{*}}{\partial t^{*}}+u \cdot \Delta T^{*}=\nabla^{2} T^{*} \\
\frac{\partial s^{*}}{\partial t^{*}}+u \cdot \Delta S^{*}=\frac{\operatorname{Pr}}{S c} \nabla^{2} S^{*}
\end{gathered}
$$

where $u=(u, w)^{T}$ is the velocity vector; $\boldsymbol{k}$ is the unit vector in the z-direction, i.e. $k=(0,1)^{T} ; p$ is the 
dynamic pressure; $\nabla, \nabla$. And $\nabla^{2}$ denote gradient, divergence and Laplace operator, respectively, that is,

$$
\nabla=\left(\frac{\partial}{\partial x}, \frac{\partial}{\partial z}\right)^{T} \quad \nabla .=\frac{\partial}{\partial x}+\frac{\partial}{\partial z} \quad \nabla^{2}=\frac{\partial^{2}}{\partial x^{2}}+\frac{\partial^{2}}{\partial z^{2}}
$$

The important dimensionless governing parameters that emerges from the above equations are thermal Rayleigh number $\left(\mathrm{Ra}_{\mathrm{T}}\right)$, salinity Rayleigh number $\left(\mathrm{Ra}_{\mathrm{S}}\right)$, Prandtl number $(\mathrm{Pr})$ and Schmidt number $(\mathrm{Sc})$ and density stability ratio $\mathrm{R}_{0}$ and they are defined as,

$$
R a_{T}=\frac{g \beta_{T} \Delta T H^{3}}{v k_{T}} ; \quad R a_{S}=\frac{g \beta_{S} \Delta S H^{3}}{v k_{T}} ; \quad P r=\frac{v}{k_{T}} ; \quad S c=\frac{v}{k_{S}}
$$

and $R_{\rho}$ is defined as $R_{p}=R a_{T} / R a_{S} ; \mathrm{k}_{\mathrm{T}}$ and $\mathrm{k}_{\mathrm{S}}$ are the thermal and salt diffusivity; $\boldsymbol{g}$ is the acceleration due to gravity and $v$ is the kinematic viscosity of the fluid, $H$ is the total height of the computational domain. $\Delta T$ and $\Delta S$ are the temperature and concentration difference between the top and bottom layers. The boundary and initial conditions are same as that in laboratory experiments presented above. The fingers form at the interface due to diffusion of heat. The computational domain was discretized into small control volumes. The set of non-dimensional governing equations (1) through (4) are integrated over each control volume. The finite volume method and Semi Implicit Method for Pressure-Linked Equations (SIMPLER) algorithm (Patankar, 1980) is used for the discretization and solutions of these equations. The equations were split with the use of Alternate Direction Implicit (ADI) method and solutions are obtained by marching in time.

Grid independence study was conducted for different Rayleigh numbers. At highest $R a_{T}\left(=3.23 \times 10^{9}\right)$ the numbers of grids used are 901:401 (x:z) for domain size of aspect ratio 1:1. The code was run on COMPAQ Alpha server ES40 having 4 CPUs per server with $667 \mathrm{MHz}$ speed. A convergence level of the order of $10^{-6}$ and $10^{-8}$ for residuals of the $u, v$ and $T$ was achieved at the high and the low Rayleigh numbers respectively. Detailed validation with experiments and published literature has been reported by Singh (2006) and Sreenivas et al., (2003, 2009).

\section{Results and discussion}

There is a wide separation in the time scales for the diffusion of the species and the momentum (Sc 1000); similarly between the diffusion length scale and the size of the physical domain. Due to these reasons, equations are stiff and are computationally expensive. Hence, while studying the effect of viscosity $(v)$ on the width of the finger, we have carried out the simulations only up to the time when fingers are just developed at the interface. Effect of viscosity on the width of the fingers is shown in Figure 4. Note that for the simulations with viscosities $\left(\mathrm{v}, \mathrm{m}^{2} / \mathrm{s}\right) 1 \times 10^{-6}, 1 \times 10^{-5}$ and $4 \times 10^{-4}$, the number of fingers $\left(\mathrm{N}_{\mathrm{f}}\right)$, observed in the unit width of the computational domain, are 102,28 and 14 respectively. $\mathrm{N}_{\mathrm{f}}$ is inversely proportional to the width of fingers. We find that finger width is proportional to $v^{(1 / 3)}$. This result suggests that fingers get thicker and thicker as the fluid viscosity is increased. Thicker fingers also develop into a high aspect ratio, lengthy fingers as they can maintain their driving force due to the larger width and reduced mixing (due to high viscosity). The trend is similar to the one observed in our experiments. In Figure 5, we present a snapshot of well-developed fingers from our simulation. It clearly demonstrates the evolution of slender and ordered finger structures which are sandwiched between two convective layers similar to colonnades sandwiched between entablature in columnar basalts. It is to be noted that waviness and tilt in the fingers is due to the dynamic interactions between the neighboring fingers, which is generally seen in the columnar basalt structures.

Our numerical results indicate that finger width is inversely proportional to the $R a_{T}^{0.28}$ (Table 1). Combining this with the results presented in Figure 4, it is evident that the finger width depends not only on the viscosity $(v)$ but on the product of viscosity and diffusion coefficient of the faster diffusing component $\left(\mathrm{k}_{\mathrm{T}}\right.$ ) (denominator of $\mathrm{Ra}_{\mathrm{T}}$ ). Kantha's (1980) experiments did not produce a marked difference in the width of the fingers because he used salt-sugar system. Increase in viscosity using glycerin-water solution is compensated by a same order with the lower diffusivity of salt (compared to that for heat). In our experiments, we used heat as the faster diffusing component and hence increase in viscosity increased the width of fingers.

A simple order of magnitude argument for the thickness of the unstable layer, $\delta$, developed at the interface of two 
lava layers, just before the formation of fingers, indicates that (Sreenivas et. al., 2009) :

$$
\delta=\left[R a_{C} v k_{T}\left(\frac{\rho}{\Delta \rho g}\right)\right]^{1 / 3}
$$

where, $\operatorname{Ra}_{\mathrm{C}}$ is a constant $(\sim 650), v$ is the kinematic viscosity of lava, $\rho$ is the density of lava and $\Delta \rho$ is the initial density difference between the top and the bottom layers of lava. The width of the columnar basalt will be proportional to $\delta$ (proportionality constant being order of one). Now, width of the columnar basalt can be estimated based on the lava properties. Conversely, we can give an order of magnitude estimate for the density difference between adjacent columns, based on the width of the columnar basalt and lava properties. In Figure 6, we present the contours of the constant width columnar basalt as a function of viscosity and $\Delta \rho$. Figure 6 was obtained by assuming thermal diffusivity, $\mathrm{k}_{\mathrm{T}}$, of lava as $10^{-7} \mathrm{~m}^{2} / \mathrm{s}$ (which is a typical value). The figure indicates that a columnar basalt having a width of $0.6 \mathrm{~m}$, formed in lava which is $4 \times 10^{5}\left(\mathrm{v}=0.4 \mathrm{~m}^{2} / \mathrm{s}\right)$ times more viscous than water will have an extremely small density contrast between neighboring columns.

Due to lack of field evidences on the evolution of columnar basalts, a number of theories have been proposed on the scaling (Reiter et al. 1987, Grossenbacher and McDuffie, 1995) and ordering (Budkewitsch and Robin, 1994, Jagla and Rojo, 2002) of columnar jointing. However, the questions of what sets the column scale and drives ordering are largely remain open (Goehring and Morris, 2006). Unlike previous theories of basalt scaling with Peclet number, which is based on the advection-diffusion model (e.g. Goehring and Morris, 2006), scaling in equation (6) considers lava properties like viscosity, thermal diffusivity and density variation due to both high temperature and concentration. Further, scale selection in double-diffusive finger system is innate and it is determined by these properties (Sreenivas et al. 2009). In essence, this could provide a clue on why different scales of basalt fingers with uncanny degree of orderliness have been observed across the world.

\section{Conclusions}

In this paper we demonstrated that convection cells of high aspect ratio can develop, which are similar to the columnar basalt structures under suitable conditions. When two layers of lava, from two eruptions, form a double diffusive system in the finger-regime, high viscosity of lava becomes crucial factor in the evolution of thick basalt-fingers having high aspect ratio. Observations presented here from laboratory experiments and numerical simulations indicate that cell-width is directly proportional to the one-third power of lava viscosity and hence at high viscosity the finger thickness can be quite large. The order of magnitude analysis also suggests that the cell-width would be larger for smaller density differences between two layers. When the molten lava solidifies, the finger boundaries become a natural path for crack propagation and hence evolve into a long columnar basaltic structure. Therefore, the double-diffusive finger model can easily explain the high aspect ratio and polygonal shape of columnar basalt. Both the experimental and the numerical model are successful in explaining the observed large aspect ratio and sandwich configuration of the columnar basalt-structures. These structures can only evolve where special condition of two layer double diffusive finger convection system is satisfied. Thus, only few basaltic lava flows satisfying this condition can develop into columnar structures, and is not a default formation in all basaltic-lava flows. This explains rarity of observation of columnar joints only in limited geographical locations.

\section{Acknowledgments}

The research is supported by JNCASR and Super Computer Education and Research Center, IISc, India.

\section{References}

Budkewitsch, P. \& Robin, P.-Y. (1994). Modeling the evolution of columnar joints. J. Volcanol. Geotherm. Res, 59, 219-239. doi:10.1016/0377-0273(94)90092-2, http://dx.doi.org/10.1016/0377-0273(94)90092-2

Davaille, A. (1999). Two-layer thermal convection in miscible viscous fluids. Journal of Fluid Mechanics, 379, 223-253. doi:10.1017/S0022112098003322, http://dx.doi.org/10.1017/S0022112098003322

Griffiths, R. W. (1986). The differing effects of compositional and thermal buoyancies on evolution of mantle diapers. Physics of the Earth and Planetary Interiors, 43, 261-273. doi:10.1016/0031-9201(86)90016-6, http://dx.doi.org/10.1016/0031-9201(86)90016-6

Groisman, A. \& Kaplan, E. (1994). An experimental study of cracking induced by desiccation. Euro Phys. Letter, 25, 415-420. doi:10.1209/0295-5075/25/6/004, http://dx.doi.org/10.1209/0295-5075/25/6/004

Grossenbacher, K. A. \& McDuffie, S. M. (1995). Conductive cooling of lava: columnar joint diameter and stria width as functions of cooling rate and thermal gradient. J. Volcanol. Geotherm. Res, 69, 95-103. doi:10.1016/0377-0273(95)00032-1, http://dx.doi.org/10.1016/0377-0273(95)00032-1 
Huppert, H. E. \& Sparks, R. S. J. (1994). Double-diffusive convection due to crystallization in Magmas. Ann. Rev. Earth Planet. Sci, 12, 11-37. doi:10.1146/annurev.ea.12.050184.000303, http://dx.doi.org/10.1146/annurev.ea.12.050184.000303

Jagla, E. A. \& Rojo, A. G. (2002). Sequential fragmentation: The origin of columnar quasihexagonal patterns. Phys. Rev. E, 65, 026203. doi:10.1103/PhysRevE.65.026203, http://dx.doi.org/10.1103/PhysRevE.65.026203

Kantha, L. H. (1980). A note on the effect of viscosity on double-diffusive process. J. Geophysical Research, 85, 4398-4404. doi:10.1029/JB085iB08p04398, http://dx.doi.org/10.1029/JB085iB08p04398

Kantha, L. H. (1981). Basalt-fingers'-origin of columnar joints?. Geol. Mag., 118, 251-264. doi:10.1017/S0016756800035731, http://dx.doi.org/10.1017/S0016756800035731

Kerr, R. C., Woods, A. W., Worster, M. G., \& Huppert, H. E. (1989). Disequilibrium and macro-segregation during solidification of a binary melt. Nature, 340, 357-362. doi:10.1038/340357a0, http://dx.doi.org/10.1038/340357a0

Muller, G. (1998). Experimental simulation of basalt columns. J. Volconology and Geothermal Research, 86, 93-96. doi:10.1016/S0377-0273(98)00045-6, http://dx.doi.org/10.1016/S0377-0273(98)00045-6

Patankar S. V. (1980). Numerical heat transfer and fluid flow. Hemisphere Publishing Company.

Pauchard, L., Adda-Bedia, M., Allain, C. \& Couder, Y. (2003). Morphologies resulting from the directional propagation of fractures. Physical Review E, 67, 027103, 1-4.

Platten, J.K., Legros, J.C. (1984). Convection in Liquids. Springer, Berlin.

Reiter M., Barroll, M. W., Minier, J. \& Clarkson, G. (1987). Thermo-mechanical model for incremental fracturing in cooling lava fows. Tectonophysics, 142, 241-260. doi:10.1016/0040-1951(87)90126-0, http://dx.doi.org/10.1016/0040-1951(87)90126-0

Shen, C.Y and Veronis, G. (1997). Numerical simulation of two-dimensional salt fingers. J. of Geophysical Research, vol.102 (C10), 23131-23143. doi:10.1029/97JC01580, http://dx.doi.org/10.1029/97JC01580

Shirtcliffe, T. G. L. \& Turner J. S. (1970). Observation of the cell structure of salt fingers. Journal of Fluid Mech, 41, 707-719. doi:10.1017/S002211207000085X, http://dx.doi.org/10.1017/S002211207000085X

Shorlin K. A, de Bruyn J. R., Graham, M., \& Morris, S. W. (2000). Development and geometry of isotropic and directional shrinkage crack patterns. Physical Review E, 61, 6950-6957. doi:10.1103/PhysRevE.61.6950, http://dx.doi.org/10.1103/PhysRevE.61.6950

Singh, O. P. (2006). Dynamics of double diffusive finger convection: structures and convective fluxes. Doctoral thesis, Indian Institute of Science, Bangalore, India.

Sosman, R. B. (1916). Types of prismatic structures in igneous rocks. J. Geology, 24, 215-234. doi:10.1086/622324, http://dx.doi.org/10.1086/622324

Spry, A. (1962). The origin of columnar jointing, particularly in basalt flows. Journal of the Australian Geological Society, 8, 192-216.

Sreenivas, K. R., Singh, O. P \& Srinivasan J. (2009). On the relationship between finger width, velocity, and fluxes in thermohaline convection. Physics of Fluids, 21(2), 026601-0266015. doi:10.1063/1.3070527, http://dx.doi.org/10.1063/1.3070527

Sreenivas, K. R., Singh, O. P. \& Srinivasan, J. (2003). Is the formation of columnar basalt due to double-diffusive finger-instability?. Advances in Fluid Mechanics, ed. Alam M., Rama Govindarajan,Ramesh, O. N. and Sreenivas K. R., Proceedings of the symposium on advances in fluid mechanics, Bangalore, India, 138.

Schmitt, R. W. (1983). The characteristics of salt fingers in variety of fluid systems, including stellar interiors, liquid metals, oceans, and magmas. Phys. Fluids, 26(9), 2373-2377. doi:10.1063/1.864419, http://dx.doi.org/10.1063/1.864419

Turner, J. S. (1979). Buoyancy Effects in Fluids, Cambridge University Press. 
Table 1. Variation of number of fingers, $\mathrm{N}_{\mathrm{f}}$ with thermal Rayleigh number, $R a_{T}$ for two sets of density stability ratio. In these simulations non-dimensional parameters $\operatorname{Pr}$ and $S c$ are 7 and 700 respectively; dimensional values of viscosity, thermal diffusivity, salinity diffusion coefficients (all in $\mathrm{m}^{2} / \mathrm{s}$ ) are $1 \times 10^{-6}, 1.428 \times 10^{-7}$ and $1.428 \times 10^{-9}$ respectively. The products, $g \beta_{T} \Delta T$ and $g \beta_{S} \Delta S$ are varied to get different Rayleigh numbers and density stability ratios.

\begin{tabular}{|c|c|c|c|}
\hline Cases & $R a_{T}$ & $N_{f}$ & $R_{\rho}$ \\
\hline (a) & $3.24 * 10^{9}$ & 50 & 6.2 \\
\hline (b) & $2.29 * 10^{9}$ & 44 & 6.2 \\
\hline (c) & $1.37 * 10^{9}$ & 36 & 6.2 \\
\hline (d) & $1.05 * 10^{9}$ & 31 & 2.0 \\
\hline (e) & $8.40 * 10^{8}$ & 30 & 6.2 \\
\hline (f) & $4.52 * 10^{8}$ & 27 & 6.2 \\
\hline (g) & $2.25 * 10^{8}$ & 24 & 6.2 \\
\hline (h) & $4.44 * 10^{8}$ & 23 & 2.0 \\
\hline (i) & $2.72 * 10^{8}$ & 20 & 2.0 \\
\hline (j) & $1.46 * 10^{8}$ & 18 & 2.0 \\
\hline (k) & $5.66 * 10^{7}$ & 16 & 6.2 \\
\hline (l) & $7.32 * 10^{7}$ & 15 & 2.0 \\
\hline (m) & $1.83 * 10^{7}$ & 10 & 2.0 \\
\hline (n) & $5.70 * 10^{6}$ & 8 & 6.2 \\
\hline (l) & $1.80 * 10^{6}$ & 6 & 2.0 \\
\hline
\end{tabular}
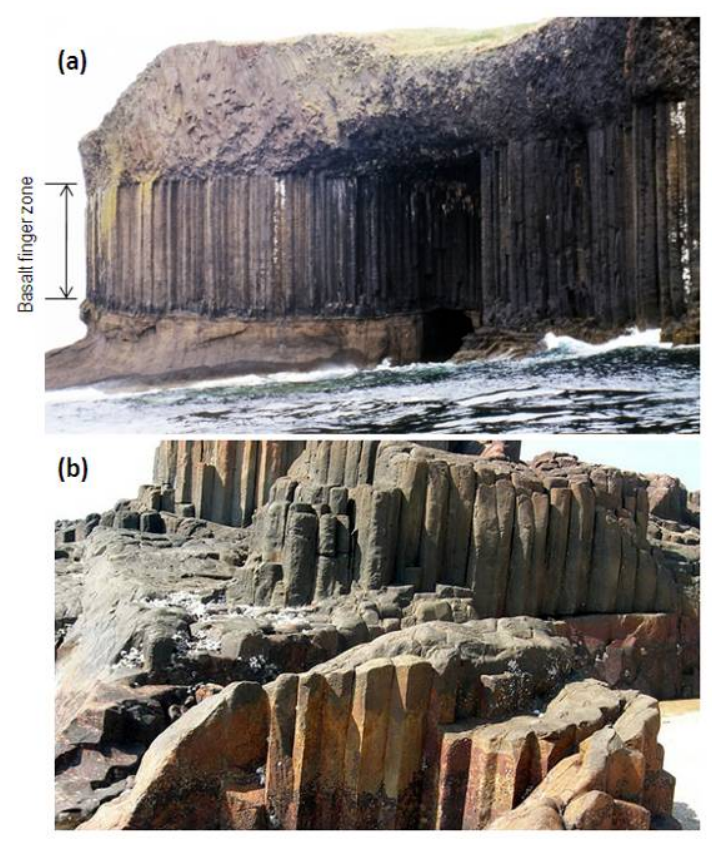

Figure 1. Some of the famous columnar basalt structures found around the world: (a) Fingal cave, Scotland, an example for sandwich configuration and (b) St. Marry's Island near Mangalore, India. Apart from the these locations shown in the figure there are other examples, Giant Causeway, Ireland; Millstream falls, Australia; Massif Central, France and many others 


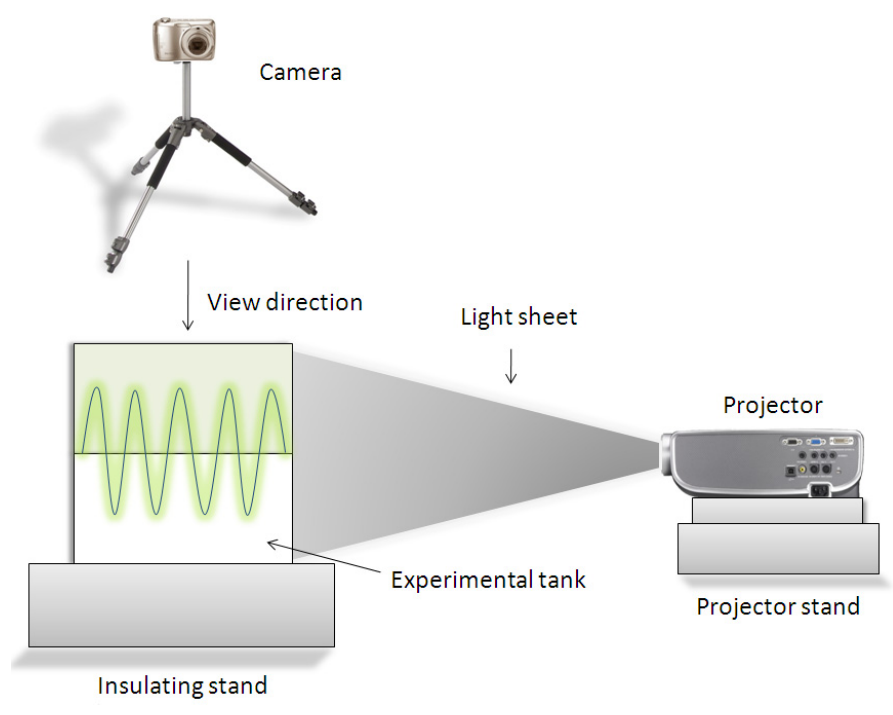

Figure 2. Schematic diagram of the experimental setup

(a)

Adiabatic wall
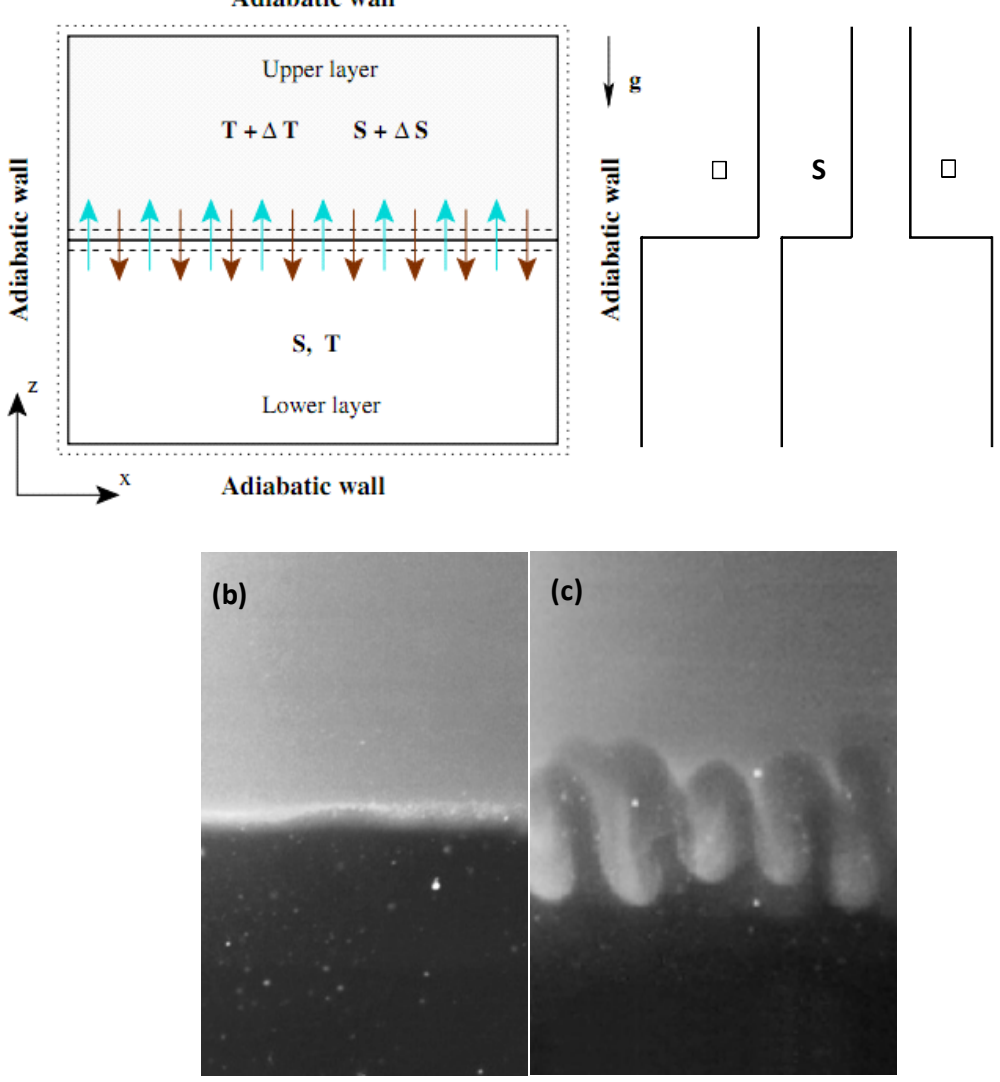

Figure 3 (a). Schematic diagram indicating the distribution of initial temperature and salinity in the upper and the lower layers of a double diffusive system. The initial profiles of temperature, salinity and density is shown in right side, (b) Flat interface between the two layers just after starting an experiment. Top fluid (white-region) contains $8 \mathrm{gm} / 1$ salt $(\Delta \mathrm{S}=8 \mathrm{gm} / \mathrm{l})$ and is $50^{\circ} \mathrm{C}$ hotter $\left(\Delta \mathrm{T}=50^{\circ} \mathrm{C}\right)$ than the bottom fluid. The darker-region is cold and fresh. The viscosity of the fluid in the both layers is same $(688 \mathrm{cP})$. Various non-dimensional parameters are $\operatorname{Pr}=4910, \mathrm{Ra}_{\mathrm{T}}=3.32 \times 10^{6}, \mathrm{Ra}_{\mathrm{S}}=2.12 \times 10^{6}$ and $\mathrm{R}_{p}=1.56$, (c) Formation of evenly spaced, thick fingers at the interface, 20 minutes after the start of the experiment 


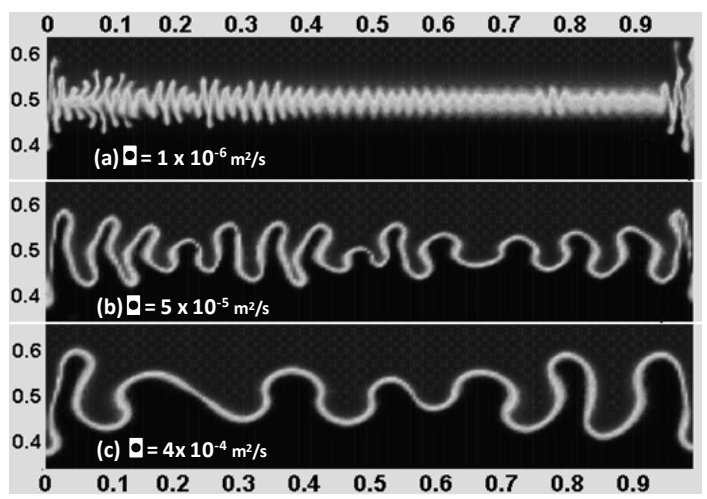

Figure 4. Result from the numerical simulation indicating the effect of viscosity ( $v)$ on the width of fingers in the same computational domain. Finger structures for three cases of viscosity are shown, (a) $v=1 \times 10^{-6} \mathrm{~m}^{2} / \mathrm{s}$ (b) $v=5 \times 10^{-5} \mathrm{~m}^{2} / \mathrm{s}$ and (c) $v=4 \times 10^{-4} \mathrm{~m}^{2} / \mathrm{s}$

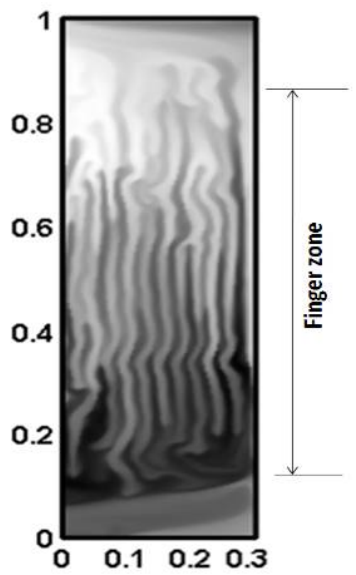

Figure 5. Well developed fingers from the numerical simulations indicating, high aspect ratio $(\sim 30)$ fingers and sandwiched structure similar to the columnar-basalt

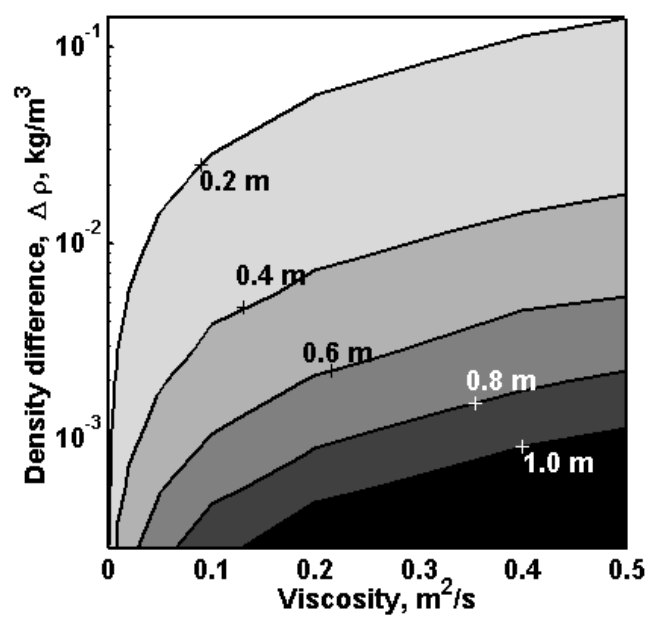

Figure 6. Plot indicating the estimated widths of columnar basalt as a function of lava viscosity (v) and initial density difference, $\Delta \rho$, between two lava layers which give rise to columnar basalt 